\title{
Teilung (Division) von Zahlen jeder Größe
}

Ein besonderer Vorteil beim Teilen mit diesen Tabellen liegt darin, daß zwei Stellen des Teilungsergebnisses (Quotient) zugleich ermittelt werden, und daß auch die Vervielfachung (Multiplikation) mit zwei Stellen zugleich erfolgt. Die Anwendung der Tabellen beim Teilen mit ein-, zwei- und dreistelligen Zahlen ergibt sich aus den nachstehenden Beispielen $1-3$ sowie den Beispielen 4 und 8 zur Anleitung, Seite V. Ist der Teiler (Divisor) größer als dreistellig, dann ist er - entgegengesetzt dem Verfahren bei der Vervielfachung (Multiplikation) - von links nach rechts in Gruppen zu drei Zahlen einzuteilen. Zur Feststellung, wie oft der Teiler (Divisor) in der zu teilenden Zahl (Dividendus) enthalten ist, sind die ersten drei Zahlen des Teilers mit der entsprechenden ersten Gruppe der zu teilenden Zahl (Dividendus) in Betracht zu zichen. Die Vervielfachung (Multiplikation) geschieht in der Weise, daß von den Ergebnissen der einzelnen Gruppen - der Einteilung des Teilers folgend - soviel Zahlen niederzuschreiben sind, wie die vervielfachte Gruppe desselben Zahlen enthält. Die übriggebliebenen Zahlen sind dem Ergebnis der nächsten Gruppe hinzuzuzählen.

\begin{tabular}{|c|c|c|c|c|}
\hline \multicolumn{5}{|c|}{ Beis } \\
\hline 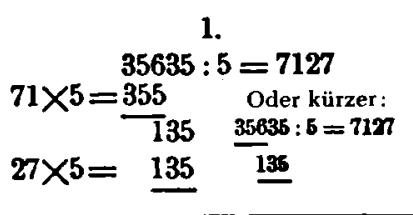 & \multicolumn{2}{|c|}{$\begin{array}{l}\frac{2 .}{270359: 37=7307} \\
73 \times 37=\frac{2701}{259} \\
07 \times 37=\underset{(0 \text { beim Ergebnis beachten })}{\frac{259}{3}}\end{array}$} & \multicolumn{2}{|c|}{$\begin{array}{c}3 . \\
87 \times 145=\frac{1262370: 145=8706}{870} \\
06 \times 145=\underline{870} \quad \text { (0 beim Ergebnis beachten!) }\end{array}$} \\
\hline \multicolumn{2}{|c|}{$\begin{array}{c}4 . \\
84 \times 145 \mid 2=\frac{1219783092: 145 \mid 2=840071}{12196 \mid 8} \\
0071 \times 145 \mid 2=\frac{\left.10309\right|^{2}}{\text { (00 beim Ergebnis beachten!) }}\end{array}$} & \multicolumn{3}{|c|}{$\begin{array}{c}5 . \\
24 \times 142|145| 37=\frac{34555539447: 142|145| 37=2431}{341|488| 88} \\
31 \times 142|145| 37=\frac{4406|506| 47}{406} 47\end{array}$} \\
\hline $\begin{array}{c}6 . \\
\begin{array}{l}1,185669: 0,141 \text { umg } \\
1185,669: 141=8,40 \\
8,4 \times 141= \\
09 \times 1184,4 \\
1269\end{array} \text { (0 beachten }\end{array}$ & $\begin{array}{r}7 \\
0,2842 \\
2,842 \\
19 \times 145=\frac{2755}{87} \\
60 \times 145=87\end{array}$ & & 960 & $\begin{aligned} 8 . \\
\\
32359,362: 1453,7 \text { umgew..: } \\
22 \times 145 \mid 37=\frac{323593,62: 145 \mid 37=22,26}{319814} \\
26 \times 145 \mid 37=\frac{377962}{37962} \\
\end{aligned}$ \\
\hline
\end{tabular}

\section{Beispiele für Stückzahl-, Zinsen- und Prozentberechnungen}

\begin{tabular}{|c|c|c|}
\hline 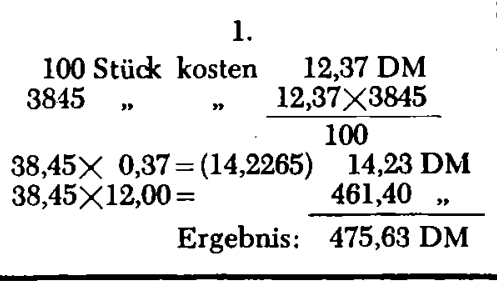 & 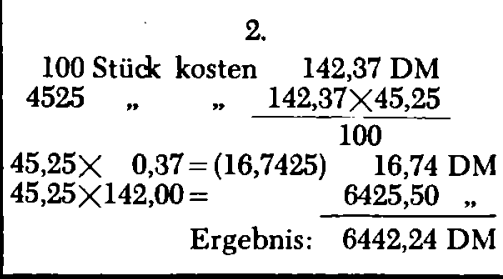 & $\begin{array}{c}3 . \\
\text { Wieviel Zinsen bringen } 345 \mathrm{DM} \\
\text { zu 41/2 Prozent in 125 Tagen? } \\
\text { Formel: Prozent } \times \text { Kapital } \times \text { Tage } \\
\frac{9 \times 345}{2 \times 125}=\frac{360 \times 100}{2 \times 360 \times 100}=\frac{38125}{72000} \\
\text { Ergebnis: } 5,39 \mathrm{DM}\end{array}$ \\
\hline 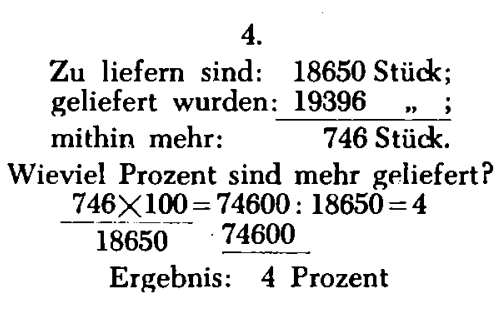 & $\begin{array}{l}5 . \\
\text { Zu liefern sind: } 24500 \text { Stück; } \\
\text { geliefert wurden: } 23226 \quad \Rightarrow ; \\
\text { mithin weniger: } \frac{1274 \text { Stück. }}{\text { Wieviel Proz. sind weniger geliefert? }} \\
\quad \frac{1274 \times 100=1274: 245=5,2}{24500} 1274,0 \\
\text { Ergebnis: } 5,2 \text { Prozent }\end{array}$ & $\begin{array}{c}6 . \\
1 \mathrm{~kg} \text { Papier kostet } 1,50 \mathrm{DM} ; \\
1 \mathrm{qm} \text { wiegt } 110 \mathrm{Gramm} \text {. } \\
\text { Wieviel kosten } 1000 \text { Bogen in Größe } \\
\text { von } 45 \times 68 \mathrm{~cm} ? \\
1000 \text { Bogen }=45 \times 68 \times 1000 \\
=3060000 \mathrm{qcm} \text { oder } 306 \mathrm{qm} ; \\
1000 \text { Bg.wiegen } 306 \times 110 \mathrm{~g}=33,660 \mathrm{~kg} ; \\
1000 \text { Bg. kost. } 33,660 \times 1,50=50,49 \mathrm{DM} \\
\text { Ergebnis: } 50,49 \mathrm{DM}\end{array}$ \\
\hline
\end{tabular}

\section{Beispiele für das Rechnen mit Brüchen}

\begin{tabular}{|c|c|c|c|}
\hline 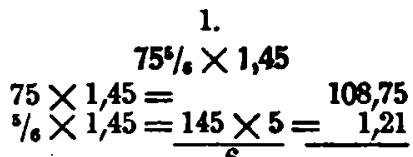 & $\begin{array}{c}2 \\
1,41: 9 / 7 \\
\frac{1,41 \times 7}{3}=\frac{987}{3}\end{array}$ & $\begin{array}{c}3 \\
15 \% \times 121 / 7 \\
\frac{85 \times 85}{6 \times 7}=\frac{8075}{42}\end{array}$ & $\begin{array}{c}\frac{4}{653 / 4: 151 \%} \\
\frac{263 \times 9}{4 \times 136}=\frac{2367}{544}\end{array}$ \\
\hline $\begin{array}{c}6 \\
\text { Ergebnis: }\end{array} \quad 109,96$ & Ergebnis: 3,29 & Ergebnis: $\quad 192^{11} / 12$ & Ergebnis: $\left.4^{191} / 544^{\star}\right)$ \\
\hline
\end{tabular}

*) Zum Kürzen von Brüchen wird empfohlen: F. Triebels „Teilbar durch?“ - Tafeln zur Feststellung der Teilbarkeit der Zahlen bis 1000 nebst Verzeichnis der unteilbaren Zahlen (Primzahlen) bis 10 000. Geh. DM 1,50. 